1

\title{
Loss of Neutralizing Antibody Response to mRNA Vaccination against SARS-CoV-2 Variants: Differing Kinetics and Strong Boosting by Breakthrough Infection
}

John P. Evans ${ }^{1,2,3}$, Cong Zeng ${ }^{1,2}$, Claire Carlin, Gerard Lozanski ${ }^{5}$, Linda J. Saif ${ }^{6,7,8}$, Eugene M. Oltz ${ }^{9}$, Richard J. Gumina ${ }^{4}$, and *Shan-Lu Liư ${ }^{1,2,8,9}$

${ }^{1}$ Center for Retrovirus Research, The Ohio State University, Columbus, OH 43210, USA

2Department of Veterinary Biosciences, The Ohio State University, Columbus, OH 43210, USA

${ }^{3}$ Molecular, Cellular, and Developmental Biology Program, The Ohio State University, Columbus, $\mathrm{OH} 43210$, USA

${ }^{4}$ Department of Internal Medicine, Division of Cardiovascular Medicine, The Ohio State University, Columbus, $\mathrm{OH} 43210$, USA

${ }^{5}$ Department of Pathology, The Ohio State University, Columbus, OH 43210, USA ${ }^{6}$ Center for Food Animal Health, Animal Sciences Department, OARDC, College of Food, Agricultural and Environmental Sciences, The Ohio State University, Wooster, OH 44691, USA ${ }^{7}$ Veterinary Preventive Medicine Department, College of Veterinary Medicine, The Ohio State University, Wooster, OH 44691, USA ${ }^{8}$ Viruses and Emerging Pathogens Program, Infectious Diseases Institute, The Ohio State University, Columbus, $\mathrm{OH} 43210$, USA ${ }^{9}$ Department of Microbial Infection and Immunity, The Ohio State University, Columbus, $\mathrm{OH}$ 43210, USA

*Corresponding Author: Liu.6244@osu.edu 


\section{Abstract:}

The waning efficacy of SARS-CoV-2 vaccines combined with the continued emergence of

33 variants resistant to vaccine-induced immunity has reignited debate over the need for booster

34 vaccines. To address this, we examined the neutralizing antibody ( $\mathrm{nAb}$ ) response against four

35 major SARS-CoV-2 variants—D614G, Alpha (B.1.1.7), Beta (B.1.351), and Delta (B.1.617.2)—in

36 health care workers (HCWs) at pre-vaccination, post-first and post-second mRNA vaccine dose,

37 and six months post-second mRNA vaccine dose. Neutralizing antibody titers against all variants,

38 especially the Delta variant, declined dramatically from four weeks to six months post-second

39 mRNA vaccine dose. Notably, SARS-CoV-2 infection enhanced vaccine durability, and mRNA-

401273 vaccinated $\mathrm{HCW}$ s also exhibited $\sim 2$-fold higher $\mathrm{nAb}$ titers than BNT162b2 vaccinated HCWs.

41 Together these results demonstrate possible waning of protection from infection against SARS-

42 CoV-2 Delta variant based on decreased nAb titers, dependent on COVID-19 status and the 43 mRNA vaccine received. 


\section{Introduction:}

Since its emergence in late 2019, the COVID-19 pandemic has led to over 252 million confirmed cases and over 5 million deaths as of November 14, 2021 (1). In response, several vaccines have been developed against SARS-CoV-2, the causative agent of COVID-19, including two novel mRNA vaccines, Moderna mRNA-1273 and Pfizer/BioNTech BNT162b2. These highly effective vaccines have helped to stem COVID-19 hospitalizations and deaths. However, the rapid evolution of SARS-CoV-2, combined with waning vaccine efficacy, remain a threat to public health.

Following its introduction into the human population, several SARS-CoV-2 variants of concern (VOCs) have emerged. Very soon after zoonotic transmission, SARS-CoV-2 acquired a predominant D614G mutation in its spike (S) protein. This mutation leads to enhanced transmissibility, likely due to increased stability of the $S$ protein, increased viral titers in the nasopharynx, and increased infectivity (2). As a result, nearly all currently circulating SARS-CoV2 strains bear the D614G mutation (3). However, as greater proportions of the world population acquired immunity against SARS-CoV-2, through infection or vaccination, new VOCs emerged that had reduced susceptibility to antibody-mediated immune responses and continued to become more transmissible $(4,5)$. One VOC, Alpha (B.1.1.7), is characterized by N-terminal domain (NTD) deletions and a key N501Y mutation in its receptor-binding domain (RBD). Alpha exhibited

62 enhanced transmissibility and rapidly spread from Europe to other parts of the world (6). Another

63 VOC to emerge at about the same time was Beta (B.1.351), which is characterized by other NTD

64 mutations and deletions, as well as key RBD mutations, including K417N, E484K, and N501Y.

65 While the Beta variant did not disseminate as widely as Alpha, it harbored strong resistance to 66 vaccine-induced immunity (7). Finally, Delta (B.1.617.2) is responsible for the most recent wave 67 of the COVID-19 pandemic and is characterized by new NTD alterations, together with key RBD 68 mutations (L452R and T478K). Delta has led to an alarming number of vaccine breakthrough 69 infections worldwide and has prompted debate about the need for vaccine booster doses. 
The extent to which the rise in breakthrough infections is caused by increased resistance

71 to vaccine-induced immunity in these variants and/or to waning durability of immunity and efficacy

72 of vaccines in preventing infection remains unclear. Reports from India, where the population was

73 still pursuing mass vaccination efforts, show minor differences in breakthrough infection rates

74 between Alpha and Delta. Specifically, BNT162b2 efficacy against symptomatic infection was

75 reported to drop from $93.4 \%$ against Alpha to $87.9 \%$ against Delta (8). However, reports from the

76 U.S. indicate that vaccine efficacy of BNT162b2 against Delta infection declined from 93\% one

77 month after vaccination to $53 \%$ at four months (9), consistent with an overall waning of vaccine

78 efficacy over time (10). A critical goal of this study is to better understand how the durability of

79 vaccine efficacy contributes to rates of breakthrough infections, especially in the context of

80 evolving SARS-CoV-2 variants. Such insights will improve strategies for allocation of booster

81 doses, recommendations for immunocompromised patients, and could guide any reformulation

82 of future SARS-CoV-2 booster doses.

To address these issues, we examined neutralizing antibody (nAb) levels in 48 vaccinated

84 health care workers (HCWs) against the major SARS-CoV-2 variants using serum collected pre-

85 vaccination, one month after the first dose of BNT162b2 or mRNA-1273, and one and six months

86 after the second dose of vaccine. Indeed, prior studies have shown that neutralizing antibody

87 levels are a major correlate for protection from SARS-CoV-2 infection (11).

89 Results:

We produced lentiviral pseudotypes expressing a Gaussia luciferase reporter gene and

91 bearing SARS-CoV-2 spike derived from D614G, Alpha, Beta, or Delta (Fig. 1A). Pseudotyped

92 virus infectivity was then determined by infection of HEK293T-ACE2 cells. Gaussia luciferase

93 secreted into the media of infected cells was assayed to determine the infectivity of produced

94 lentiviral pseudotypes. We did not find significant differences in pseudotyped lentivirus infectivity 
95 for the four variants (all containing D614G) tested (Fig. 1B), despite some reports of drastically 96 increased transmission and spread for some VOCs, especially the Delta variant (12).

We used our previously reported $(13,14)$ highly-sensitive SARS-CoV-2 pseudotyped lentivirus-based virus neutralization assay to assess nAb titers in HCW samples collected under approved IRB protocols (2020H0228 and 2020H0527). The 48 HCW samples included 22 mRNA1273 and 26 BNT162b2 vaccinated individuals, with a median age of 37 years (IQR = 31.7543.25). Samples were collected from HCWs with median time points of 222 days (IQR $=215-$ 225.75) pre-first vaccine dose (Pre), 21 days (IQR = 19.25-23) post-first vaccine dose (Post 1st), 26 days $(I Q R=22.5-28)$ post-second vaccine dose (Post 2 nd $)$, and 194 days $(I Q R=190=197.75)$ post-second vaccine dose (Six Months). According to the titer of pseudotyped viruses, we adjusted the volumes of each so that equivalent infectious viruses were used in neutralization assays. HCW serum samples underwent 4-fold serial dilutions followed by the addition of pseudotyped virus for one hr neutralization, with final dilutions of 1:80, 1:320, 1:1280, 1:5120, 1:20480, and no serum control. HEK293T-ACE2 cells were then infected with neutralized virus and Gaussia luciferase activity was assayed $48 \mathrm{hrs}$ and $72 \mathrm{hrs}$ after infection. Neutralizing titer $50 \%\left(\mathrm{NT}_{50}\right)$ values were determined by least-squares fit, non-linear regression in GraphPad Prism 5.

We compared the strength of the nAb titers over time against all four variants tested.

113 Following the first dose of mRNA vaccine, a strong nAb response was induced among HCWs 114 compared to pre-vaccination across all variants $(p<0.001)$, which efficiently blocked virus entry;

115 this was despite the huge variation in nAb titers of these individuals including against D614G $116($ mean $=1140,95 \% \mathrm{Cl}=317-1963$, range $=100-15954)($ Fig. 1C). However, across all variants, 117 between $14.6 \%$ (7/48) and 45.8\% (22/48) of HCWs exhibited $\mathrm{NT}_{50}$ values below detection limit $118\left(\mathrm{NT}_{50}<100\right)$ following the first dose of vaccine (Fig. 1C-F). These initial nAb titers fell to $0.0 \%$ $119(0 / 48)$ to $4.2 \%(2 / 48)$ for all variants following a second vaccine dose, with a 2-3-fold increase in 120 mean nAb titers compared to the first dose $(p<0.001)$ (Fig. 1C-F). Notably, four HCWs with 
121 higher nAb titers after the first vaccine dose did not show an increase, but a plateau or slight 122 decline in nAb titers following the second dose (Fig. 1C-F). These four individuals included one 123 that was anti-SARS-CoV-2-N positive at pre-vaccination, and three that were anti-N positive post124 first vaccine dose-indicating infection either prior to or shortly after their first vaccine dose. We 125 found that, following two vaccine doses, the Alpha, Beta, and Delta VOCs exhibited a 1.3- ( $p<$ 126 0.001), 3.2- $(p<0.001)$, and 2.2-fold ( $p<0.001)$ lower $\mathrm{NT}_{50}$ values compared to D614G, 127 respectively (Fig. 1C-F). Critically, six months post-vaccination, there was a 3.5-10.7-fold 128 reduction in nAb levels against all variants examined, with $37.5 \%(18 / 48)$ to $56.3 \%(27 / 48)$ of 129 HCWs exhibiting $\mathrm{NT}_{50}$ levels below the limit of detection (Fig. 1C-F). The mean $\mathrm{NT}_{50}$ values for 130 Alpha, Beta, and Delta variants at six months were 1.3-, 1.7-, and 3.6-fold lower than that of 131 D614G, respectively, although the differences in these low nAb titer groups were not statistically significant (Fig. 1C-F).

We also examined the correlation between time post-second dose and $\log _{10}$ transformed

$134 \mathrm{NT}_{50}$ values. We found a statistically significant association between these values for all four variants (Fig. 1G-J). This corresponded to an approximately 10-fold decline in $\mathrm{NT}_{50}$ for D614G, Alpha, and Delta $\left(R^{2}=0.0452-0.594, p<0.0001\right)$ every $\sim 22$ weeks compared with Beta $\left(R^{2}=\right.$ 0.286, $\mathrm{p}<0.001$ ) every $\sim 37$ weeks (Fig. 1G-J). the $48 \mathrm{HCW}$ s examined, one was anti-SARS-CoV-2 $\mathrm{N}$ positive by ELISA pre-vaccination, four were anti-N positive at their post-first vaccine dose sample, three at their post-second vaccine dose sample, and four at their six-month vaccine sample-indicating that these 12 subjects were

142 infected by SARS-CoV-2 at different phases of vaccination (Fig. 2A). At the time of pre143 vaccination sample collection D614G was the major circulating SARS-CoV-2 variant, while at the 144 time off post-first dose and post-second dose D614G and Alpha were circulating, and at the six 145 month time point Delta was the dominant strain. Notably, not all patients remained anti-N positive, 146 but were still considered to have been infected for the purpose of analysis. Following the first 
147 vaccine dose, anti-N positive HCWs exhibited 11.7-fold higher mean $\mathrm{NT}_{50}(\mathrm{p}<0.001)$ against all

148 four viruses compared to the anti-N negative HCWs (Fig. 2B). This difference diminished to 2.3-

149 fold following a second vaccine dose $(p<0.001)$ (Fig. 2B). However, at six months post-

150 vaccination, anti-N positive HCWs exhibited 6.1-fold higher $\mathrm{NT}_{50}$ values than anti-N negative

151 HCWs for all variants $(p=0.042)$ (Fig. 2B). Interestingly, we found that the differences in $N_{50}$

152 between anti-N positive and negative HCWs were greater and more statistically significant for

153 D614G and Alpha compared with the Beta and Delta variants, likely due to the strong

154 neutralization resistance of the latter VOCs (Fig. 2C). Notably, for anti-N negative HCWs,

155 between $41.7 \%$ (15/36) and 66.7\% (24/36) of subjects exhibited $\mathrm{NT}_{50}$ against all four variants that

156 were below detection limit at six months, in sharp contrast to anti-N positive individuals, who were

157 between $8.3 \%(1 / 12)$ and $25.0 \%$ (3/12) (Fig. 2C).

We further examined the difference in nAb durability between Moderna mRNA-1273 and

Pfizer/BioNTech BNT162b2 vaccinated HCWs. Across all variants over the full-time course, we

observed that mRNA-1273 elicited an overall 2.2-fold higher nAb response than the BNT162b2

$(p<0.001)$ (Fig. 2D). In particular, following two vaccine doses, mRNA-1273 vaccinated HCWs

exhibited 2.1-, 2.3-, 2.4-, and 1.3-fold higher nAb response compared to BNT162b2-vaccinated with $18.2 \%(4 / 22)$ to $36.4 \%(8 / 22)$ of mRNA-1273-vaccinated HCWs falling below detection limit for the four variants compared to $53.8 \%(14 / 26)$ to $73.1 \%$ (19/26) for BNT162b2 (Fig. 2E).

We examined additional factors that may contribute to strength and duration of the nAb response to vaccination, including age and sex. We observed no significant correlation for age and $\mathrm{NT}_{50}$ against $\mathrm{D} 614 \mathrm{G}$ at any time point (Fig. 3A-C), potentially influenced by our relatively younger pool of study subjects. However, male HCWs exhibited significantly higher $\mathrm{NT}_{50}$ titers

171 compared to females (Fig. 3D). 


\section{Discussion:}

In summary, we report a dramatic decline of SARS-CoV-2 nAb at six months post-mRNA

175 vaccination and examined several key factors accounting for these kinetics. Most critically, we

176 observed a drastic drop in nAb titers from 3-4 weeks to six months post-second vaccine dose,

177 with more than $50 \%$ of $\mathrm{HCW}$ exhibiting $\mathrm{NT}_{50}$ values below detection limit against Delta at the

178 latter time. This number increased to almost $70 \%$ for the anti-N negative HCWs, which was in

179 sharp contrast to that of anti-N positive HCWs, with $25 \%$ below the background. Thus, additional

180 antigen exposures are necessary to improve the durability of the SARS-CoV-2 nAb response,

181 consistent with data from administration of mRNA vaccine booster doses (16). Together, these

182 results support a rationale for the need for boosters and alternative vaccination strategies to

183 achieve long-term protection from infection with SARS-CoV-2.

Additionally, we observed that individuals vaccinated with BNT162b2 exhibited lower nAb

titers than individuals vaccinated with mRNA-1273. However, the trend for declining nAb titers

was consistent for both vaccines. Thus, both mRNA vaccines require booster doses to maintain

protective $n A b$ levels, although the waning of $n A b$ responses likely occurs over a relatively longer

period of time for mRNA-1273. Further examination of the durability of cellular immunity following mRNA vaccination is needed, as this more persistent immunity may limit the rates of hospitalization and death, which remain low for mRNA vaccinated individuals (17).

In this study, we found that all three VOCs consistently had reduced $\mathrm{NT}_{50}$ values compared

192 to D614G at all time points, with Beta showing the most pronounced nAb resistance, followed by

193 Delta. These results are consistent with preliminary reports from ours and other groups (14, 18,

194 19). However, we found that the Delta variant exhibited comparable or even higher resistance to

195 nAbs than Beta for samples collected at six months post vaccination. The more modest drop in

$196 \mathrm{NT}_{50}$ values at six months for the Beta variant was unclear, but likely the result of this variant's

197 pre-existing strong resistance to neutralization following the second dose of vaccination. Further,

198 the more dramatic decline in nAb titers against Delta could be attributed to a lower frequency and 
durability of neutralizing antibody-producing plasma cells. As reported by others, the rampant spread of Delta in vaccinated and unvaccinated populations is likely related to other factors such as its high replication kinetics and transmissibility (20) coupled with its comparable neutralization resistance.

Materials and Methods:

Health Care Worker Cohort: approved IRB protocols (2020H0228 and 2020H0527). These $48 \mathrm{HCW}$ ranged in age from 2261 years $($ median $=37 ; \mathrm{IQR}=31.75-43.25)$ and included 26 male and 22 female HCWs. HCWs were vaccinated with either Moderna mRNA-1273 $(n=22)$ or Pfizer/BioNTech BNT162b2 $(n=$ 26). Sera were collected from HCWs at 4 time points, with median time points being 222 days

$211(I Q R=215-225.75)$ pre-first vaccine dose (Pre), 21 days (IQR = 19.25-23) post-first vaccine dose

$212\left(\right.$ Post $\left.1^{\text {st }}\right), 26$ days $(I Q R=22.5-28)$ post-second vaccine dose $\left(\right.$ Post $\left.2^{\text {nd }}\right)$, and 194 days $(I Q R=$ $213190=197.75)$ post-second vaccine dose (6 Months). HCWs received their second vaccine dose 214 between January and February of 2021.

217 anti-N positive for their post-first vaccine dose sample, three for their post-second vaccine dose 218 sample, and four for their six-month vaccine sample-indicating that these 12 subjects were 219 infected by SARS-CoV-2 at the different phases of vaccination.

\section{Constructs for Pseudotyping Virus Production:}

Production of lentiviral pseudotyped virus was performed using a previously reported 223 protocol using pNL4-3-HIV-1-inGluc vector $(13,14,21-23)$. This vector is a pNL4-3-HIV-1 $\Delta$ Env

224 construct and contains a Gaussia luciferase reporter gene with a CMV promoter both oriented in 
an anti-sense orientation relative to the HIV-1 genome. This Gaussia luciferase reporter gene then contains a sense orientation intron, which prevents expression of Gaussia luciferase in the virus producing cells. However, after the intron is spliced from full length virus genomes and upon

228 integration into target cells, target cells can produce Gaussia luciferase, which is secreted in 229 mammalian cell culture (24). Constructs encoding $\mathrm{N}$ - and C-terminal flag-tagged SARS-CoV-2 230 spike (S) for each variant — D614G, Alpha (B.1.1.7), Beta (B.1.351), and Delta (B.1.617.2) 231 were synthesized and cloned into pcDNA3.1 vector using Kpnl/BamHI restriction enzyme cloning by GenScript BioTech (Piscataway, NJ).

Cell Lines and Maintenance:

HEK293T cells (CRL-11268, CVCL_1926, ATCC, Manassas, VA) and HEK293T-ACE2 cells (NR-52511, BEI Resources, ATCC, Manassas, VA) were maintained in Dulbeco's Modified Eagles Medium (Gibco, 11965-092, ThermoFisher Scientific, Waltham, MA) supplemented with

$23810 \%(\mathrm{v} / \mathrm{v})$ fetal bovine serum (F1051, Sigma-Aldrich, St. Louis, MO) and 1\% (v/v) penicillin/streptomycin (SV30010, HyClone Laboratories Inc., Logan, UT). Cells were maintained in at $37^{\circ} \mathrm{C}$ and $5 \% \mathrm{CO}_{2}$.

244 HIV-1-inGluc and pcDNA3.1 vector expressing the spike of interest (D614G, B.1.1.7, B.1.351, or $\mathrm{hrs}$, and $72 \mathrm{hrs}$ after transfection, then was pooled and stored at $-80^{\circ} \mathrm{C}$.

248 SARS-CoV-2 variants were used to infect HEK293T-ACE2 cells. Then, 48 hrs and 72 hrs after 249 infection, Gaussia luciferase activity in the media of infected cells was determined. $20 \mu \mathrm{L}$ of cell 250 culture media and $20 \mu \mathrm{L}$ of Gaussia luciferase substrate (0.1M Tris (T6066, MilliporeSigma, 
251 Burlington, MA) pH 7.4, 0.3M sodium ascorbate (S1349, Spectrum Chemical Mfg. Corp., New

252 Brunswick, NJ), $10 \mu \mathrm{M}$ coelenterazine (CZ2.5, GoldBio, St. Louis, MO)) were combined in a white 253 polystyrene 96-well plate. Luminescence was immediately measured by a BioTek Cytation5 plate-

254 reader.

Virus Neutralization Assays:

Virus neutralization assays were performed as previously reported $(13,14,23)$. In a 96well format, HCW serum was 4-fold serial diluted and $100 \mu \mathrm{L}$ of pseudotyped virus was added

259 (final dilutions of 1:80, 1:320, 1:1280, 1:5120, 1:20480, and no serum). Note that, to ensure 260 comparable results between SARS-CoV-2 variants, equivalent amounts of infectious virus were 261 used based on the pre-determined virus titers. The virus was incubated with HCW serum for $1 \mathrm{hr}$ at $37^{\circ} \mathrm{C}$, followed by infection of HEK293T-ACE2 cells seeded on a 96-well polystyrene tissue culture plate. Gaussia luciferase activity in cell culture media was then assayed $48 \mathrm{hrs}$ and $72 \mathrm{hrs}$

264 after infection as described above. Neutralizing titer 50\% (NT50) for each serum sample was determined by non-linear regression with least squares fit in GraphPad Prism 5 (GraphPad Software, San Diego, California).

Anti-N ELISA was performed as previously reported (13). ELISA was performed by using 270 the EDI Novel Coronavirus COVID-19 N protein IgG ELISA Kit (KT-1032, EDI, San Diego, CA)

271 following manufacturer's protocol. Briefly, $100 \mu \mathrm{L}$ of a 1:100 dilution of HCW serum was added to 272 microplates coated with SARS-CoV-2 neucleocapsid $(\mathrm{N})$ antigen and incubated for $30 \mathrm{~min}$. Plates 273 were then washed and treated with $100 \mu \mathrm{L}$ of HRP labeled anti-human-IgG antibody (31220, EDI,

274 San Diego, CA) for $30 \mathrm{~min}$. Then plates were washed and $100 \mu \mathrm{L}$ of ELISA HRP substrate (10020,

275 EDI, San Diego, CA) was added and incubated for 20 min before $100 \mu \mathrm{L}$ of stop solution (10030, 
276 EDI, San Diego, CA) was added. Absorbance at $450 \mathrm{~nm}$ was read by spectrophotometric plate

277 reader using Gen 5 software.

Statistical Analyses:

Statistical analysis was done with GraphPad Prism 5. Comparisons between multiple groups were done using one-way ANOVA with Bonferroni's multiple testing correction (Figs. 1A, 2B) or one-way repeated measures ANOVA with Bonferroni's multiple testing correction (Figs. 1C-F). For comparisons between two "treatments" across multiple groups, a two-way ANOVA

284 with Bonferroni's multiple testing correction was used (Figs. 2C and 2E). For comparisons 285 between two groups, an unpaired, two-tailed student's t-test with Welch's correction was used 286 (Figs. 2D, S1D). For correlative analyses between two continuous variables, a linear regression model with least squares fit was used with $\log _{10}$ transformed $\mathrm{NT}_{50}$ values to better approximate linearity (Figs. 1G-J, S1A-C).

Competing Interests:

291 The authors declare no competing interest.

\section{Funding Statement:}

294 This work was supported by a fund provided by an anonymous private donor to OSU; additional 295 support of S.-L.L.'s lab includes an NIH grant R01 Al150473. J.P.E. was supported by Glenn 296 Barber Fellowship from the Ohio State University College of Veterinary Medicine. S.-L.L., R.J.G., 297 L.J.S. and E.M.O. were supported by the National Cancer Institute of the NIH under award no. 298 U54CA260582. The content is solely the responsibility of the authors and does not necessarily 299 represent the official views of the National Institutes of Health. R.J.G. was additionally supported 300 by NIH R01 HL127442-01A1 and the Robert J. Anthony Fund for Cardiovascular Research, and 301 L.J.S. was partially supported by NIH R01 HD095881. 


\section{Author Contributions:}

304 J.P.E. conducted neutralization assays, analyzed data, and drafted the manuscript. C.Z. aided in 305 neutralization assays, review of manuscript, and provided valuable discussion and insight. C.C. 306 contributed to recruitment of HCWs and sample collection. R.J.G. contributed to study design, 307 provided HCW samples and subject information, reviewed the manuscript, and provided valuable 308 discussion and insight. G.L. provided anti-N ELISA data. S.-L.L. contributed to study design, 309 directed laboratory personnel, and aided in drafting and revision of the manuscript. C.C., G.L., 310 L.J.S., E.M.O., and R.J.G. provided valuable discussion and revision of the manuscript.

\section{Acknowledgements:}

313 We thank David Derse, Marc Johnson, Fang Li, and Ali Ellebedy for providing plasmids, cells, 314 and antibodies. We thank members of Liu lab for sharing reagents and discussion. We also thank 315 the NIH AIDS Reagent Program and BEI Resources for supplying important reagents that made 316 this work possible. We thank the Clinical Research Center/Center for Clinical Research 317 Management of The Ohio State University Wexner Medical Center and The Ohio State University 318 College of Medicine in Columbus, Ohio, specifically Francesca Madiai, Claire Carlin, Dina 319 McGowan, Breona Edwards, Evan Long, and Trina Wemlinger, for logistics, collection and 320 processing of samples.

\section{References:}

1. World Health Organization. COVID-19 weekly epidemiological update, 16 November 2021. 2021.

2. Plante JA, Liu Y, Liu J, Xia H, Johnson BA, Lokugamage KG, et al. Spike mutation D614G alters SARS-CoV-2 fitness. Nature. 2021;592(7852):116-21.

3. Korber B, Fischer WM, Gnanakaran S, Yoon H, Theiler J, Abfalterer W, et al. Tracking changes in SARS-CoV-2 Spike: evidence that D614G increases infectivity of the COVID-19 virus. Cell. 2020;182(4):812-27. e19.

4. Abdool Karim SS, de Oliveira T. New SARS-CoV-2 variants-clinical, public health, and vaccine implications. New England Journal of Medicine. 2021;384(19):1866-8. 
332

333

334

335

336

337

338

339

340

341

342

343

344

345

346

347

348

349

350

351

352

353

354

355

356

357

358

359

360

361

362

363

364

365

366

367

368

369

370

371

372

373

374

375

376

377

378

379

380

381

382

5. Singh J, Rahman SA, Ehtesham NZ, Hira S, Hasnain SE. SARS-CoV-2 variants of concern are emerging in India. Nature medicine. 2021:1-3.

6. Washington NL, Gangavarapu K, Zeller M, Bolze A, Cirulli ET, Barrett KMS, et al. Emergence and rapid transmission of SARS-CoV-2 B. 1.1. 7 in the United States. Cell. 2021;184(10):2587-94. e7.

7. Zhou D, Dejnirattisai W, Supasa P, Liu C, Mentzer AJ, Ginn HM, et al. Evidence of escape of SARS-CoV-2 variant B. 1.351 from natural and vaccine-induced sera. Cell. 2021.

8. Bernal JL, Andrews N, Gower C, Gallagher E, Simmons R, Thelwall S, et al. Effectiveness of COVID-19 vaccines against the B. 1.617. 2 variant. medRxiv. 2021.

9. Tartof SY, Slezak JM, Fischer H, Hong V, Ackerson BK, Ranasinghe ON, et al. Effectiveness of mRNA BNT162b2 COVID-19 vaccine up to 6 months in a large integrated health system in the USA: a retrospective cohort study. The Lancet. 2021.

10. Mizrahi B, Lotan R, Kalkstein N, Peretz A, Perez G, Ben-Tov A, et al. Correlation of SARSCoV-2-breakthrough infections to time-from-vaccine. Nature Communications. 2021;12(1):1-5.

11. Khoury DS, Cromer D, Reynaldi A, Schlub TE, Wheatley AK, Juno JA, et al. Neutralizing antibody levels are highly predictive of immune protection from symptomatic SARS-CoV-2 infection. Nature medicine. 2021:1-7.

12. Campbell $F$, Archer $B$, Laurenson-Schafer $H$, Jinnai $Y$, Konings $F$, Batra N, et al. Increased transmissibility and global spread of SARS-CoV-2 variants of concern as at June 2021. Eurosurveillance. 2021;26(24):2100509.

13. Zeng C, Evans JP, Pearson R, Qu P, Zheng Y-M, Robinson RT, et al. Neutralizing antibody against SARS-CoV-2 spike in COVID-19 patients, health care workers, and convalescent plasma donors. JCl insight. 2020;5(22).

14. Zeng C, Evans JP, Faraone JN, Qu P, Zheng Y-M, Saif L, et al. Neutralization of SARSCoV-2 Variants of Concern Harboring Q677H. Mbio. 2021;12(5):e02510-21.

15. Anichini G, Terrosi C, Gandolfo C, Gori Savellini G, Fabrizi S, Miceli GB, et al. SARS-CoV2 antibody response in persons with past natural infection. New England Journal of Medicine. 2021.

16. Barda N, Dagan N, Cohen C, Hernán MA, Lipsitch M, Kohane IS, et al. Effectiveness of a third dose of the BNT162b2 mRNA COVID-19 vaccine for preventing severe outcomes in Israel: an observational study. The Lancet. 2021.

17. Scobie HM, Johnson AG, Suthar AB, Severson R, Alden NB, Balter $S$, et al. Monitoring incidence of covid-19 cases, hospitalizations, and deaths, by vaccination status-13 US jurisdictions, April 4-July 17, 2021. Morbidity and Mortality Weekly Report. 2021;70(37):1284.

18. Planas D, Veyer D, Baidaliuk A, Staropoli I, Guivel-Benhassine F, Rajah MM, et al. Reduced sensitivity of SARS-CoV-2 variant Delta to antibody neutralization. Nature. 2021:1-7.

19. Liu C, Ginn HM, Dejnirattisai W, Supasa P, Wang B, Tuekprakhon A, et al. Reduced neutralization of SARS-CoV-2 B. 1.617 by vaccine and convalescent serum. Cell. 2021;184(16):4220-36. e13.

20. Mlcochova P, Kemp SA, Dhar MS, Papa G, Meng B, Ferreira IA, et al. SARS-CoV-2 B. 1.617. 2 Delta variant replication and immune evasion. Nature. 2021:1-6.

21. Mazurov D, Ilinskaya A, Heidecker G, Lloyd P, Derse D. Quantitative comparison of HTLV1 and HIV-1 cell-to-cell infection with new replication dependent vectors. PLoS pathogens. 2010;6(2).

22. Yu J, Li M, Wilkins J, Ding S, Swartz TH, Esposito AM, et al. IFITM proteins restrict HIV-1 infection by antagonizing the envelope glycoprotein. Cell reports. 2015;13(1):145-56.

23. Zeng C, Evans JP, Reisinger S, Woyach J, Liscynesky C, El Boghdadly Z, et al. Impaired Neutralizing Antibody Response to COVID-19 mRNA Vaccines in Cancer Patients. Medrxiv. 2021. 
24. Goerke AR, Loening AM, Gambhir SS, Swartz JR. Cell-free metabolic engineering promotes high-level production of bioactive Gaussia princeps luciferase. Metabolic engineering. 2008;10(3-4):187-200.

\section{Figure 1: The durability of vaccine-induced immunity wanes over time, with a virtual loss}

at six months for the Delta variant. Gaussia luciferase reporter gene containing lentivirus pseudotypes were produced bearing the spike (S) protein from SARS-CoV-2 variants. (A) Schematic representations of the SARS-CoV-2 variant spikes tested are shown which contain the indicated mutations. These include D614G, Alpha (B.1.1.7), Beta (B.1.351), and Delta (B.1.617.2). The schematics highlight the location of the $\mathrm{S} 1$ and $\mathrm{S} 2$ subunits as well as the $\mathrm{N}$-terminal domain (NTD), receptor binding domain (RBD), fusion peptide (FP), and transmembrane region (TM). (B) Lentivirus pseudotypes were used to infect HEK293T-ACE2 cells and 48hrs after infection media was harvested from infected cells and assayed for Gaussia luciferase activity to determine the relative infectivity of variant pseudotyped virus. (C-F) Lentivirus pseudotyped with SARS-CoV-2 S from D614G (C), Alpha (D), Beta (E), and Delta (F) were incubated for $1 \mathrm{hr}$ to neutralize with serial dilutions (1:80, 1:320, 1:1280, 1:5120, 1:20480, and no serum) of health care worker (HCW) serum collected pre vaccination, post vaccination with a first dose of Pfizer/BioNTech BNT162b2 or Moderna mRNA-1273, post vaccination with a second dose of mRNA vaccine, and six months post vaccination with a second dose of mRNA vaccine. Neutralized virus was then used to infect HEK293T-ACE2 cells and Gaussia luciferase activity was assayed $48 \mathrm{hrs}$ and $72 \mathrm{hrs}$ after infection. Neutralization titers $50 \%\left(\mathrm{NT}_{50}\right)$ were determined by least-squares fit non-linear regression. Mean $\mathrm{NT}_{50}$ are shown at the top of the plots, and $\mathrm{NT}_{50}$ values below 100 were considered background. (G-J) $\log _{10}$ transformed (to better approximate linearity) $\mathrm{NT}_{50}$ values against D614G (G), Alpha (H), Beta (I), and Delta (J) variants were plotted against days postsecond vaccine dose of sample collection. The equation of the fitted curve, the goodness of fit $\left(R^{2}\right)$, and p-value for the curve are displayed on each plot. The dotted lines correspond to the background level $\left(\mathrm{NT}_{50}<100\right)$. Statistical significance was determined by one-way ANOVA with 
Bonferroni's correction (B), one-way repeated measures ANOVA with Bonferroni's correction (CF), or by least-squares fit linear regression (G-J). In call cases, ${ }^{*} p<0.05$; ${ }^{* *} p<0.01 ;{ }^{* * *} p<0.001$; ns: not significant.

$416 \mathrm{~N}$ positive $\left(\mathrm{OD}_{450}>0.4\right.$ at any time point; $\left.\mathrm{n}=12\right)$ and $\mathrm{HCW}$ who never became anti-N positive

$417\left(\mathrm{OD}_{450}<0.4\right.$ for all time points; $\left.\mathrm{n}=36\right)$. $(\mathbf{B}, \mathbf{C}) \mathrm{HCW}$ were divided by prior COVID-19 status as 418 determined by anti-SARS-CoV-2 N ELISA. HCWs with anti-N above the cut-off value of 0.4 for 419 any time point $(\mathrm{n}=12)$ were considered as COVID-19 positive during the study period. $\mathrm{NT}_{50}$ 420 values against all four variants combined (B) or separated (C) for anti-N positive HCWs are 421 compared to anti-N negative HCWs for samples collected post first mRNA vaccine dose, post second mRNA vaccine dose, and six months post second mRNA vaccine dose, respectively. (D,

427 significance was determined by one-way ANOVA with Bonferroni's correction (B) two-way, 428 repeated measures ANOVA with Bonferroni's correction (C, E) or unpaired two-tailed t-test with Welch's correction (D). In call cases, ${ }^{*} p<0.05$; ${ }^{* *} p<0.01$; ${ }^{* * *} p<0.001$; ns: not significant.

431 Figure 3: Impact of age and sex on response to mRNA vaccination. (A-C) $\log _{10}$ transformed

$432 \mathrm{NT}_{50}$ titers against D614G-SARS-CoV-2 S pseudotyped lentivirus are plotted against age (in 433 years) at time of second vaccine dose for HCW samples collected post-fist vaccine dose (A), 434 post-second vaccine dose (B), and 6 months post-second vaccine dose (C). (D) $\mathrm{NT}_{50}$ values 435 against all variants at all time points were compared for male and female HCWs, with mean $\mathrm{NT}_{50}$ 
bioRxiv preprint doi: https://doi.org/10.1101/2021.12 06.471455. this version posted December 7,2021. The copyright holder for this preprint

(which was not certified by peer review) is the author/funder, who has granted bioRxiv a license to display the preprint in perpetuity. It is made available under aCC-BY-NC 4.0 International license.

436 values displayed at the top of the plot. Statistical significance was determined by linear regression

437 with least squares residual fit (A-C) or by unpaired, two-tailed student's t-test with Welch's

438 correction (D). P-values are indicated as 'ns' (not significant) for $p>0.05$ or * $p<0.05,{ }^{* *} p<0.01$, $439{ }^{* * *} p<0.001$

440 


\section{A}

D614G

(which was not certified by peer review) is the author/funder, who has granted bioRxiv $\longleftarrow \mathrm{S}_{1} \longrightarrow$ akailable under aC $82 \mathrm{BY}-\mathrm{NC} 4.0$ InterpatiBal license.

\section{D614G}

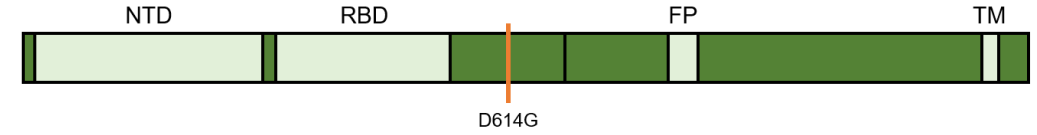

Alpha

(B.1.1.7)

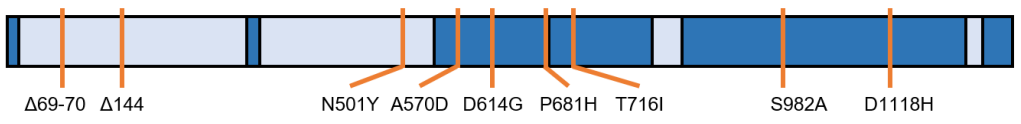

Beta

(B.1.351)

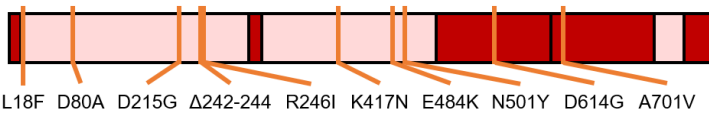

Delta

(B.1.617.2)
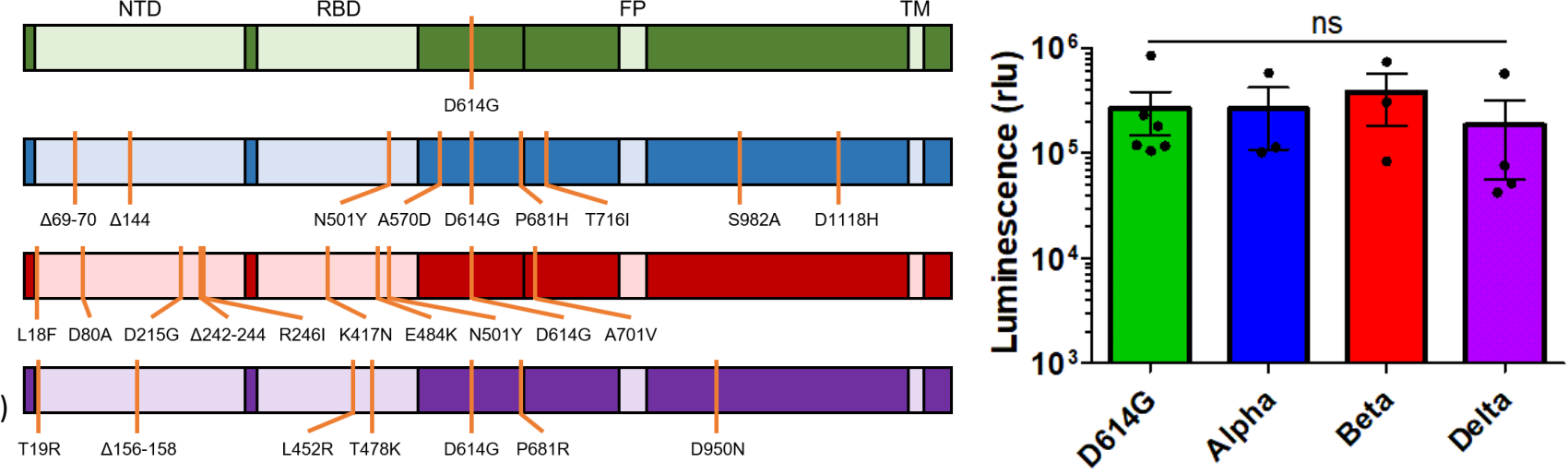

\section{C}

C D614G

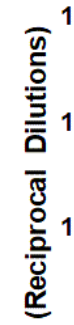

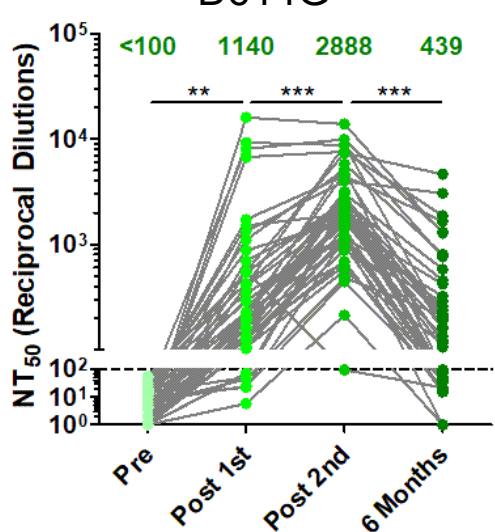

D Alpha

G
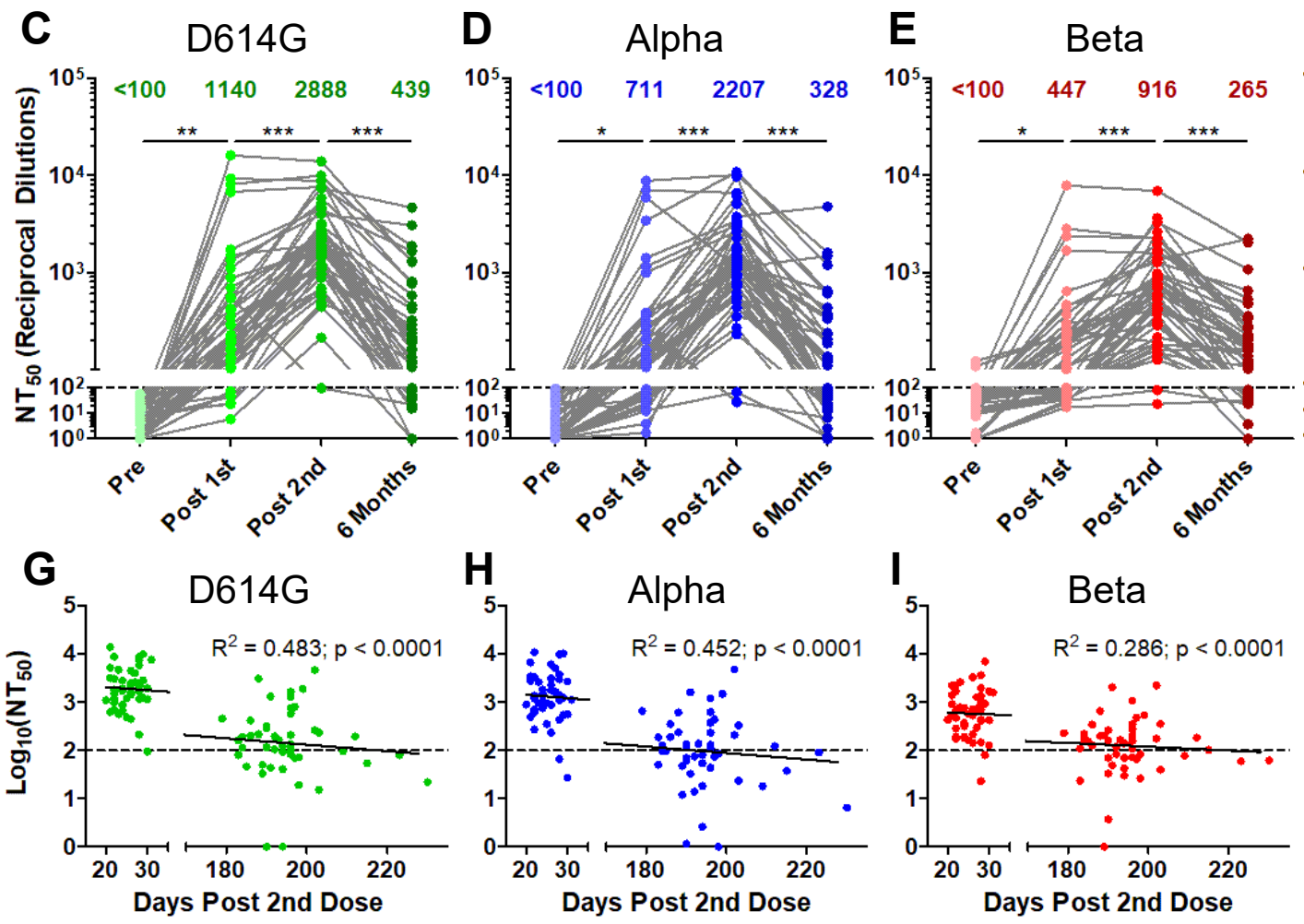

F Delta
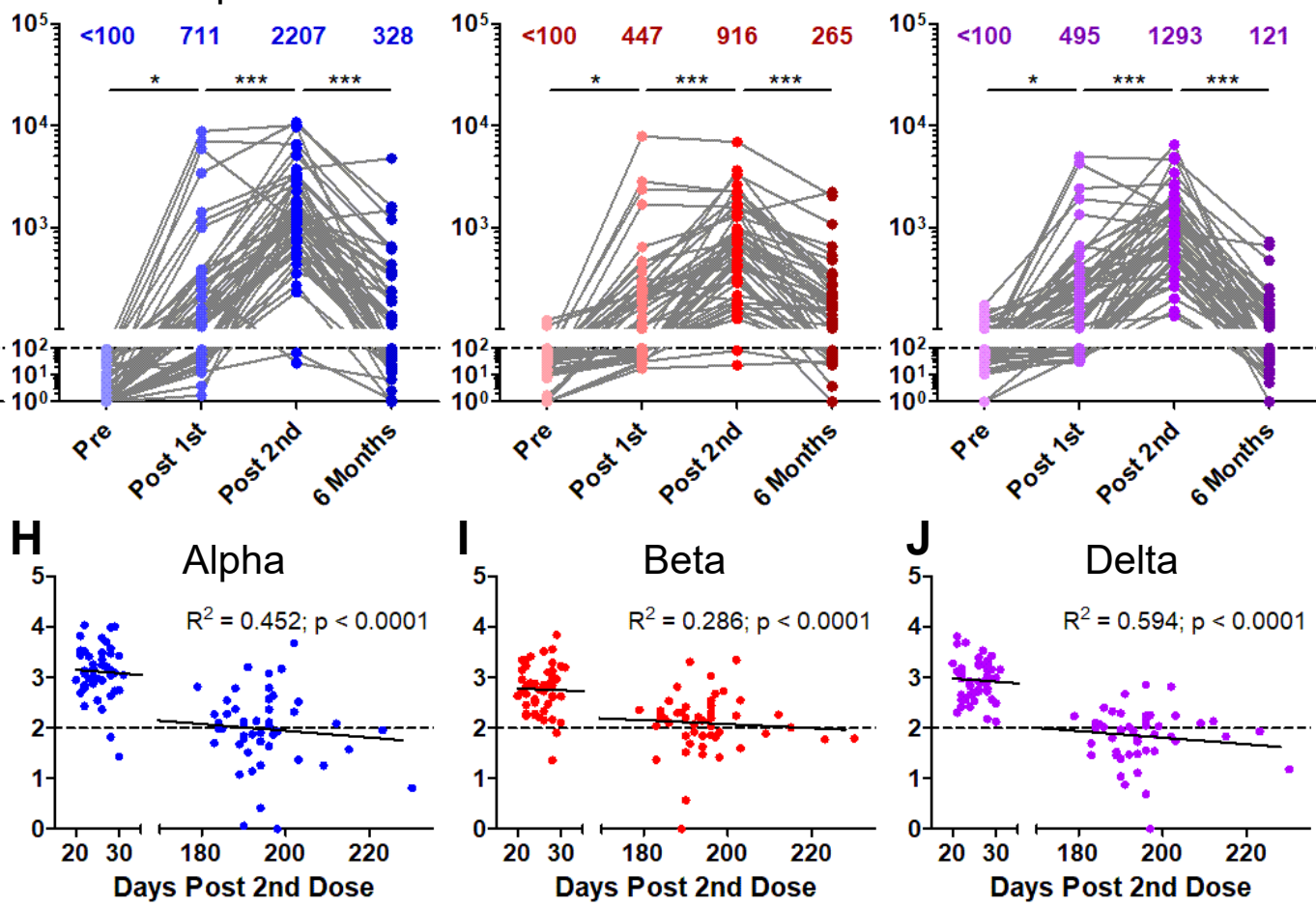
